\title{
Lemierre's Syndrome: Not Just a Sore Throat
}

\author{
Mary Henry ${ }^{1}$, Kanwaljit Singh ${ }^{2}$, Sowjanya Potturu ${ }^{3}$
}

\begin{abstract}
Lemierre's syndrome is a rare condition, sharing a symptom spectrum with some other viral diseases which can sometimes mask the diagnosis of the syndrome. It is important to keep a low threshold of the possibility of the syndrome, especially in someone who develops features of systemic upset after a sore throat. If undiagnosed and left untreated the syndrome can be fatal and can have severe multiorgan complications requiring various forms of medical and surgical interventions. We present the case of Lemierre's syndrome in a 32-year-old man who was successfully treated during an inpatient stay involving intensive care unit, followed by a community-based course of antimicrobial therapy for several weeks. Keywords: Dysphagia, Lemierre's syndrome, Oropharyngeal infection, Septic phlebitis, Septicemia, Thrombosis.

Journal of Postgraduate Medicine, Education and Research (2021): 10.5005/jp-journals-10028-1447
\end{abstract}

\section{Case Description}

We present the case of a 32-year-old Caucasian man coming to the emergency department with a 5-day history of sore throat, neck, and right ear pain along with fever and shortness of breath. On arrival, he was tachypneic (respiratory rate of 26 breaths per minute) and tachycardic (heart rate of 118), with a temperature of $40^{\circ} \mathrm{C}$. Examination of his throat showed congestion, redness, and swollen tonsils. Examination of the chest revealed basal crackles. His general practitioner (GP) had started him on penicillin $V$ on the same day prior to his presentation to the hospital. In the hospital, he quickly became more unwell with a drop in oxygen saturations to $88 \%$ on room air on pulse oximetry, systolic blood pressure of $<90 \mathrm{~mm} \mathrm{Hg}$, and heart rate of 140 beats per minute and persistently high temperature of $>38^{\circ} \mathrm{C}$. He was given immediate medical support in the form of intravenous antibiotics, intravenous fluids, and oxygen and transferred to the intensive care unit on site. He was a smoker and did not have any significant medical history. A computed tomography pulmonary angiography (CTPA) revealed enlarged mediastinal lymph nodes and solid lung nodules predominantly in the right lower lobe, suspicious of underlying malignancy or atypical pneumonia; along with straightening of the interventricular septum suspicious of early right heart strain. An echocardiogram performed later showed no evidence of right heart strain. Initial laboratory investigations revealed raised inflammatory markers with C-reactive protein (CRP) values at 295, white cell count 12.4, neutrophils 10.7. There was an evidence of previous Epstein-Barr virus (EBV) infection, negative Legionella serology, and negative cytomegalovirus (CMV). A further computed tomography (CT) scan of the neck and thorax showed a large right parapharyngeal abscess causing thrombophlebitis of the right lingual vein, a tributary of the internal jugular vein (Fig. 1 shows highlighted area of the parapharyngeal abscess). The lung lesions previously seen had progressed with further lesions in the upper lobe, in keeping with septic emboli (Fig. 2 shows highlighted areas of septic emboli). A diagnosis of Lemierre's syndrome was made. Following discussion with hematology specialists, treatment dose low-molecular-weight heparin was prescribed and the patient was referred to ear, nose, and throat (ENT) specialists.
${ }^{1}$ Department of Ophthalmology, Russell Hall Hospital, Dudley, West Midlands, UK

${ }^{2,3}$ Department of Healthcare for Older People, Good Hope Hospital, Sutton Coldfield, West Midlands, UK

Corresponding Author: Kanwaljit Singh, Department of Healthcare for Older People, Good Hope Hospital, Sutton Coldfield, West Midlands, UK, Phone: +44 1214247738, e-mail: kanwaljit.singh@nhs.net

How to cite this article: Henry M, Singh K, Potturu S. Lemierre's Syndrome: Not Just a Sore Throat. J Postgrad Med Edu Res 2021;55(4):180-182.

Source of support: Nil

Conflict of interest: None

Blood cultures were negative on admission. However, aspiration from the right peritonsillar region grew anaerobic cocci on culture and antibiotic therapy was rationalized as advised by the microbiology specialists. The patient was listed for further aspiration and drainage in theater; however, he self-discharged before this could be done. The team were nevertheless able to

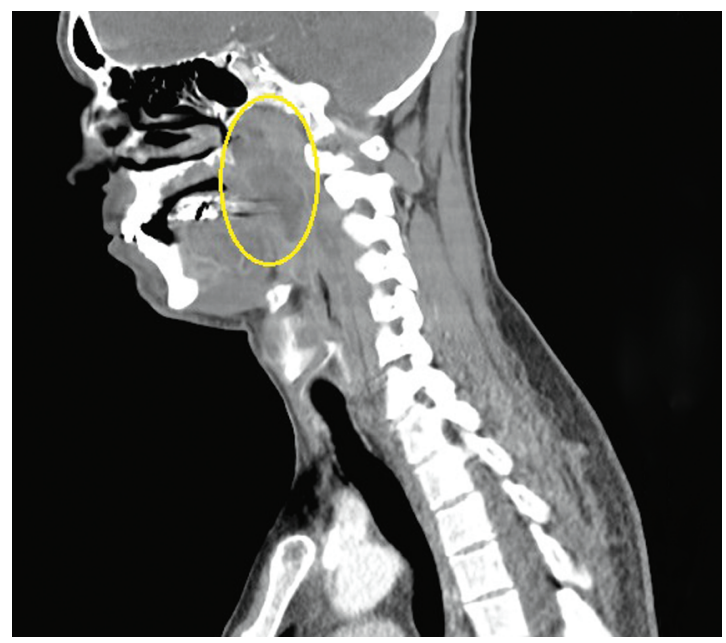

Fig. 1: Highlighted area of the parapharyngeal abscess

(-) The Author(s). 2021 Open Access This article is distributed under the terms of the Creative Commons Attribution 4.0International License (https://creativecommons. org/licenses/by-nc/4.0/), which permits unrestricted use, distribution, and non-commercial reproduction in any medium, provided you give appropriate credit to the original author(s) and the source, provide a link to the Creative Commons license, and indicate if changes were made. The Creative Commons Public Domain Dedication waiver (http://creativecommons.org/publicdomain/zero/1.0/) applies to the data made available in this article, unless otherwise stated. 


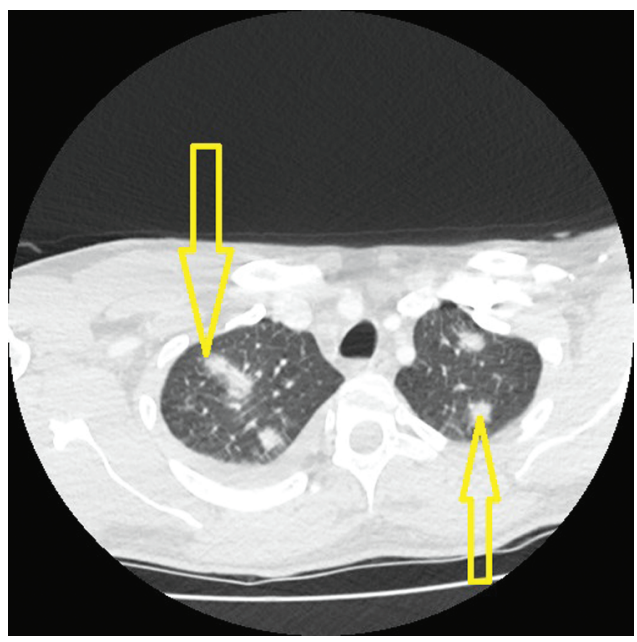

Fig. 2: Highlighted areas of septic emboli

ensure that he went home with at least 4 weeks of co-amoxiclav and metronidazole as per microbiology advice. During a follow-up telephone conversation with the patient to monitor his progress, he confirmed improvement in his overall condition.

\section{Discussion}

Septic phlebitis and thrombosis of the internal jugular vein secondary to an oropharyngeal infection, known as Lemierre's syndrome, was first described in 1936 by Andre-Alfred Lemierre. ${ }^{1,2}$ With an incidence of approximately 0.8 per million, it remains a rare condition, typically affecting young adults between the ages of 16 and 30 , with equal sex distribution. ${ }^{1-3}$ It describes opportunistic infection by commensal flora of the oral cavity and pharynx when the host's mucosal surface is damaged. A common pathogen is Fusobacterium necrophorum; an anaerobic, gram-negative bacilli, found in the first case of LS. ${ }^{2,4}$ Although Fusobacterium spp. has been found in approximately half of the cases, other known organisms include Streptococcus spp. and Staphylococcus aureus and mixed infections can also occur., ${ }^{1,2}$

The disease is characterized by bacteremia, internal jugular vein thrombosis, and the development of septic emboli. ${ }^{3}$ The lungs are a common site of disseminated infection; however, abscess formation may also occur in the liver, joint spaces, and skin. ${ }^{3}$ The organism spreads from the pharyngeal space to the internal jugular vein via the tonsillar vein by the production of endotoxin and other virulence factors including leukocidin and hemolysin. ${ }^{1-3}$

Typical presentation includes dysphagia, swelling at the mandible, neck pain, fever, and arthralgia (11-27\%). ${ }^{2,5}$ High fever and rigors often indicate septicemia and usually occur within 1 week of onset of throat symptoms. However, oropharyngeal symptoms have often resolved by the time sepsis ensues. ${ }^{1,6}$ The majority of patients have septic emboli in the lungs (79-100\%), which present with cough, hemoptysis, dyspnea, and pleuritic chest pain. ${ }^{6}$ Multiple nodular infiltrates and small pleural effusions can be seen on chest $\mathrm{X}$-rays. These lesions can rapidly progress despite antibiotic therapy and empyema develops in 10 to $15 \% .^{3}$

Although the diagnosis of Lemierre's syndrome is predominantly clinical, baseline laboratory tests assist in diagnosis. ${ }^{6}$ Patients often display leukocytosis and neutrocytosis along with elevated liver enzymes and acute phase reactants (CRP and erythrocyte sedimentation rate). ${ }^{2}$ Computed tomography imaging aids diagnosis, identifying thrombi, as the most specific sign is thrombophlebitis if the internal jugular. ${ }^{4,6}$ Diagnosis is confirmed on blood or fluid culture. However, Fusobacterium can be difficult to culture and typically takes 3 to 5 days to grow which can lead to delay in treatment if the diagnosis is not suspected clinically. ${ }^{2-4,6}$

Lemierre's syndrome can be fatal and complications of the untreated syndrome include meningitis, osteomyelitis, and endocarditis due to metastatic infection and septic emboli deposition throughout the body. If treated, mortality is $2 \%$ but a delay of 4 or more days has shown an increase from 10 to $25 \%{ }^{7}$ Therefore, prompt treatment is vital. ${ }^{1,2}$ Yet because initial symptoms can mimic less severe conditions such as tonsillitis and the common cold, diagnosis is often delayed. ${ }^{4}$ Lemierre's syndrome is often misdiagnosed as infectious mononucleosis but differentiating features include unilateral internal jugular vein thrombosis and generalized lymphadenopathy compared with mainly cervical in infectious mononucleosis. ${ }^{3}$

Treatment is guided against oral anaerobes and extended depending on the severity of the infection. ${ }^{6}$ Most causative pathogens have proven to have beta-lactamase activity giving rise to empiric treatment using beta-lactamase resistant betalactams. ${ }^{2}$ Fusobacterium spp. is $100 \%$ sensitive to metronidazole, cefotaxime, and imipenem and data suggest metronidazole may give the most rapid response; however, monotherapy with metronidazole is not advised due to infection severity and the frequency of mixed infection. ${ }^{2,3,6}$ Clindamycin has been used effectively for the treatment of lesions including septic emboli and lung abscesses. ${ }^{2}$ There is variation in course of treatment and duration has not yet been established. It ranges between 9 and 84 days and is guided by the severity of illness and response of patients to treatment. ${ }^{2,6}$ Recommendation is usually a 2-week course of a beta-lactam antibiotic, intravenously, along with metronidazole and clindamycin. ${ }^{2,4}$ This course can be extended depending on the severity of the illness. A further 4 to 6 weeks of oral antibiotic therapy is then usually given. ${ }^{1,6,7}$ Antibiotic therapy should be narrowed once susceptibilities are known. ${ }^{2}$

Anticoagulation therapy has been used by some in the management of Lemierre's syndrome; however, there is no recommended guidance regarding this as the evidence supporting it is not conclusive. There is no real consensus on the effectiveness of the use of anticoagulation or the significance of coagulopathies associated with Lemierre's syndrome. ${ }^{2,4}$

Complications such as abscesses, empyema, and septic joints must be surgically drained after starting antibiotics. ${ }^{1,2}$ Although rarely indicated, thrombectomy should be considered in cases with an intracerebral or mediastinal extension of internal jugular vein thrombi and other surgical interventions such as venous ligation or resection may be required in patients not responding to medical therapy. ${ }^{2,8,9}$

\section{CONCLUSION}

It is important to differentiate Lemierre's syndrome from other viral illnesses affecting the oropharynx and upper airways presenting with sore throat-like features. The suspected cases especially the 
ones with systemic upset-related features should be investigated promptly and a robust treatment offered accordingly.

\section{ACKnOWLedgments}

We would like to acknowledge the support of our radiology colleagues for providing us with the relevant images.

\section{INFORMED CONSENT}

Written informed consent for the paper to be published (including images, case history, and data) was obtained from the patient for publication of this paper, including accompanying images.

\section{References}

1. Charles K, Flinn WR, Neschis DG. Lemierre's syndrome: a potentially fatal complication that may require vascular surgical intervention. J Vasc Surg 2005;42(5):1023-1025. DOI: 10.1016/j.jvs.2005.07.005.

2. Pryor J, Sharma P, Reedy R. Lemierre's syndrome: a case report of a child with partial treatment of streptococcal pharyngitis. Int J Clin Pediatr 2020;9(1):4-8. DOI: 10.14740/ijcp347.
3. Riordan T, Wilson M. Lemierre's syndrome: more than a historical curiosa. Postgrad Med J 2004;80(944):328-334. DOI: 10.1136/ pgmj.2003.014274.

4. Le C, Gennaro D, Marshall D, et al. Lemierre's syndrome: one rare disease-two case studies. J Clin Pharm Ther 2019;44(1):122-124. DOI: $10.1111 /$ jcpt.12774.

5. Riordan T. Human infection with Fusobacterium necrophorum (necrobacillosis), with a focus on Lemierre's syndrome. Clin Microbiol Rev 2007;20(4):622-659. DOI: 10.1128/CMR.00011-07.

6. Lu MD, Vasavada Z, Tanner C. Lemierre syndrome following oropharyngeal infection: a case series. J Am Board Fam Med 2009;22(1):79-83. DOI: 10.3122/jabfm.2009.01.070247.

7. Johannesen KM, Bodtger U. Lemierre's syndrome: current perspectives on diagnosis and management. Infect Drug Resist 2016;9:221-227. DOI: 10.2147/IDR.S95050.

8. Sinave CP, Hardy GJ, Fardy PW. The Lemierre syndrome: suppurative thrombophlebitis of the internal jugular vein secondary to oropharyngeal infection. Medicine (Baltimore) 1989;68(2):85-94. DOI: 10.1097/00005792-198903000-00002.

9. Shadowen RD, Trevor RP. Lemierre's postanginal septicemia: internal jugular vein thrombosis related to pharyngeal infection. South Med J 1989;82(12):1583-1584. DOI: 10.1097/00007611-198912000-00034. 